\title{
A new kind info sample theorem and its aliasing error estimation
}

\author{
ZHAO Zhen-yu ${ }^{1}$, LIU Shan-cun ${ }^{2}$ \\ 1. School of Economics and Management; Ningxia University Yinchuan ;China 750021 \\ 2. School of Economics and Management; Beijing University of Aeronautics and Astronautics \\ Beijing ; China 100191
}

Keywords: Info; sample theorem; aliasing error estiamtion; improvement

\begin{abstract}
The signal must be bandlimited in its reconstructing by using the Whittaker-Shannon series, the request is too high.The paper constructs a kind of new series instead of the Whittaker-Shannon series, for a given signal,using the new series and its samples to reconstruct the signal,through this method the request of the signal can be lowered, at the same time the aliasing error estimation is given.
\end{abstract}

\section{Introduction}

In information theory, the fundamental of sampling theorem is actually the interpolation problem using exponential functions. Sampling theorem is the theoretical foundation of pulse-code modulation (PCM) of our age. And it is also one of elementary tools of signal processing, and has been researched for a long time as an active research topic internationally. Since Shannon sampling theorem was published in 1949, it has been given the high attention. Assuming that the signal is with limited bandwidth, it can be reconstructed by Whittaker-Shannon series as

$$
L_{\sigma}(f ; x)=\sum_{k \in Z} f\left(x_{k}\right) F_{\sigma}\left(x-x_{k}\right)=\frac{1}{\sigma} \sum_{k \in Z} f\left(x_{k}\right) \frac{2 \sin \frac{\sigma}{2}\left(x-x_{k}\right)}{x-x_{k}}
$$

Where $F_{\sigma}(x)=\frac{2 \sin \frac{\sigma}{2} x}{\sigma x}, \quad x_{k}=\frac{2 k \pi}{\sigma}, k \in Z$; initial signal can be constructed by formula (*) cooperating with $f\left(k \frac{2 \pi}{\sigma}\right)$, it holds on the condition that $f$ must be a limited bandwidth signal. In order to relax such a condition on $f$ which may not conform to the restriction, we propose a new series to replace Whittaker-Shannon series in the paper to reconstruct sampling signal cooperating with $f\left(k \frac{2 \pi}{\sigma}\right)$. This proposal could easy the restrictions on the reconstructed signal. Moreover, it could give the precise rank of aliasing error. It is a breakthrough in the reconstruction of

sampling signals. It may get a splendid perspective of information and mathematics.

We assume that $B(R)$ is the space of measurable functions, where $R$ is the set of real numbers. $B(R)$ is the subspace of $B(R)$ which consists of the set of functions $U_{\sigma}$ which subject to

$$
\sum_{k \in Z}\left|U_{\sigma}\left(x-\frac{2 k \pi}{\sigma}\right)\right| \sigma>0
$$

And $U_{\sigma}$ uniformly trends in the interval $[0,2 \pi] \cdot B_{\sigma}^{p}(1 \leq p<\infty, \sigma>0)$ represents the entie function with the exponential $\sigma$ ( $C$ is the complex plane, $|f(z)| \leq e^{\sigma|y|}\|f\|_{\infty}, z=x+i y \in C$ and $f \in L^{p}(R)$ ).

In fact, Whittaker-Shannon series is the same as Lagrange interpolation operator, i.e.

$$
L_{\sigma}(f ; x)=\sum_{k \in Z} f\left(x_{k}\right) F_{\sigma}\left(x-x_{k}\right)=\frac{1}{\sigma} \sum_{k \in Z} f\left(x_{k}\right) \frac{2 \sin \frac{\sigma}{2}\left(x-x_{k}\right)}{x-x_{k}} \text { Where } F_{\sigma}(x)=\frac{2 \sin \frac{\sigma}{2} x}{\sigma x}, \quad \text { the node is }
$$

$x_{k}=\frac{2 k \pi}{\sigma}, k \in Z$. However, this operator of interpolation doesn't always uniformly converge to the 
function for interpolation purpose. To overcome the shortcoming of the Lagrange interpolation operator, there are a lot of improvements of Lagrange interpolator.In 1930, at the National Conference of Math. of U.S.S.R. in Kharkov, S.N.Bernstein put forward for improving the convergence of interpolation operator, we can abandon the condition of interpolate and keep the simple construct. Naturally, what will be interesting in is how to construct the interpolation polynomial satisfying S.N.Bernstein's requirement as above so that its convergence order is the best. In 1987 a new interpolation operator is constructed by F.Pinter:

$$
U_{k \sigma}(x)=4 F_{\sigma}(x)^{3}-3 F_{\sigma}(x)^{4}
$$

Obtain the interpolation operator:

$$
U_{\sigma}(f ; x)=\sum_{k \in Z} f\left(x_{k}\right) U_{k \sigma}\left(x-x_{k}\right)
$$

Moreover, the next statements holds true:

THEOREM A: If $f$ is uniformly continuous and bounded, then

$$
\sup _{x \in R} f(x)-U_{\sigma}(f ; x) \mid=O\left(\omega\left(f, \frac{1}{\sigma}\right)\right)
$$

The paper is inspired by previous work, re-average the Whittaker-Shannon series, get a new kind Whittaker-Shannon series: $H_{\sigma}(f ; r, x)$, we try to reconstruct the signal by $H_{\sigma}(f ; r, x)$, cooperating with $f\left(k \frac{2 \pi}{\sigma}\right)$, at the same time, the aliasing error estimation is given 1 .

Let

Where $F_{k}^{(\sigma)}(x)=F_{\sigma}\left(x-x_{k}\right)=\frac{2 \sin \frac{\sigma}{2}\left(x-x_{k}\right)}{\sigma\left(x-x_{k}\right)}=\frac{(-1)^{k} 2 \sin \frac{\sigma}{2} x}{\sigma\left(x-x_{k}\right)}$

with nodes $x_{k}=\frac{2 k \pi}{\sigma}$, is the fundamental function of Whittaker-Shannon, the combination series of Whittaker-Shannon $H_{\sigma}(f ; r, x)$ is given.

$$
H_{\sigma}(f ; r, x)=\sum_{k \in Z} f\left(x_{k}\right) A_{k}^{(\sigma)}(x) .
$$

\section{improved sample theorem}

Before giving the improved sample theorem, we introduced the lemma

LEMMA 1 The following estimations are valid

$$
\sum_{k \in Z}\left|A_{k}^{(\sigma)}(x)\right|=\frac{1}{2} \sum_{k \in Z}\left|F_{k}^{(\sigma)}\left(x-\frac{\pi}{\sigma}\right)+F_{k}^{(\sigma)}\left(x+\frac{\pi}{\sigma}\right)\right|=O(1)
$$

Proof: because

$$
\begin{aligned}
\sum_{k \in Z}\left|A_{k}^{(\sigma)}(x)\right|=\frac{1}{2} \sum_{k \in Z}\left|F_{k}^{(\sigma)}\left(x-\frac{\pi}{\sigma}\right)+F_{k}^{(\sigma)}\left(x+\frac{\pi}{\sigma}\right)\right| \\
=\frac{1}{2} \sum_{k \in Z}\left|F_{\sigma}\left(x-x_{k}-\frac{\pi}{\sigma}\right)+F_{\sigma}\left(x-x_{k}+\frac{\pi}{\sigma}\right)\right| \\
=\frac{1}{2} \sum_{k \in Z}\left|\frac{2 \sin \frac{\sigma}{2}\left(x-x_{k}-\frac{\pi}{\sigma}\right)}{\sigma\left(x-x_{k}-\frac{\pi}{\sigma}\right)}+\frac{2 \sin \frac{\sigma}{2}\left(x-x_{k}+\frac{\pi}{\sigma}\right)}{\sigma\left(x-x_{k}+\frac{\pi}{\sigma}\right)}\right| \\
=2\left|\cos \frac{\sigma}{2} x\right| \sum_{k \in Z}\left|\frac{1}{\sigma x-2 k \pi-\pi}-\frac{1}{\sigma x-2 k \pi+\pi}\right|
\end{aligned}
$$

${ }^{1}$ approximation error is the aliasing error in electronic engineering 


$$
=2\left|\cos \frac{\sigma}{2} x\right| \sum_{k \in Z}\left|\frac{2 \pi}{(\sigma x-2 k \pi)^{2}-\pi^{2}}\right|
$$

Thus $\sum_{k \in Z}\left|A_{k}^{(\sigma)}(x)\right|=O(1)$

LEMMA 2 If $p(x)$ is entire function with the exponential type $\sigma$, then:

$$
B_{\sigma}(p, x)=\frac{1}{2} p\left(x-\frac{\pi}{\sigma}\right)+\frac{1}{2} p\left(x+\frac{\pi}{\sigma}\right)
$$

Proof: because

$$
B_{\sigma}(p, x)=\sum_{k \in Z} p\left(x_{k}\right) A_{k}^{\sigma}(x)
$$

Where $A_{k}^{(\sigma)}(x)=\frac{1}{2}\left(F_{k}^{(\sigma)}\left(x-\frac{\pi}{\sigma}\right)+F_{k}^{(\sigma)}\left(x+\frac{\pi}{\sigma}\right)\right)$, for the exponential type entire function coincide with its interpolation polynomials, thus:

$$
\begin{aligned}
B_{\sigma}(p, x)= & \frac{1}{2} \sum_{k \in Z} p\left(x_{k}\right) F_{k}^{\sigma}\left(x-\frac{\pi}{\sigma}\right)+\frac{1}{2} \sum_{k \in Z} p\left(x_{k}\right) F_{k}^{\sigma}\left(x+\frac{\pi}{\sigma}\right) \\
& =\frac{1}{2} p\left(x-\frac{\pi}{\sigma}\right)+\frac{1}{2} p\left(x+\frac{\pi}{\sigma}\right)
\end{aligned}
$$

THEOREM 1 If $f \in C^{j}, j \leq r$, then

$$
\left|H_{\sigma}(f ; r, x)-f(x)\right|=O\left(A_{\sigma}^{*}(f)+\frac{1}{\sigma^{j}} \omega\left(f^{(j)} ; \frac{1}{\sigma}\right)\right)
$$

\section{Proof of theorem 1}

$$
\begin{aligned}
\text { let, } \Delta_{h}^{r+1} f(x)= & \sum_{i=0}^{r+1}(-1)^{s+i}\left(\begin{array}{c}
r+1 \\
i
\end{array}\right) f(x+(i-s) h), \text { where } h=\frac{2 \pi}{\sigma}, \sigma>0 \text {, thus } \\
H_{\sigma}(f ; r, x)-f(x) & =\sum_{k \in Z} f\left(x_{k}\right) A_{k}(x)-f(x) \\
& =\frac{1}{2^{r+1}} \sum_{k=-\infty}^{+\infty} \Delta_{h}^{r+1} f\left(x_{k}\right) F_{k}^{(\sigma)}(x)+\sum_{k \in Z} f\left(x_{k}\right) F_{k}^{(\sigma)}(x)-f(x)
\end{aligned}
$$

$F(x)$ represents entire function with the exponential type $\sigma$ satisfies:

$$
|F(x)-f(x)| \leq A_{\sigma}^{*}(f)
$$

for the exponential type entire function coincide with its interpolation polynomials:

$$
\sum_{k \in Z}\left(\frac{1}{2^{r+1}} \Delta_{h}^{r+1} F\left(x_{k}\right)+F\left(x_{k}\right)\right) F_{k}^{(\sigma)}(x)=\frac{1}{2^{r+1}} \Delta_{h}^{r+1} F(x)+F(x)
$$

from(1)we can get

$$
\begin{aligned}
& H_{\sigma}(f ; r, x)-f(x) \\
& =\sum_{k \in Z}\left(\frac{1}{2^{r+1}} \Delta_{h}^{r+1}\left(f\left(x_{k}\right)-F\left(x_{k}\right)\right)+f\left(x_{k}\right)-F\left(x_{k}\right)\right) F_{k}^{(\sigma)}(x)+ \\
& {\left[\frac{1}{2^{r+1}} \Delta_{h}^{r+1}(F(x)-f(x))+F(x)-f(x)\right]+\frac{1}{2^{r+1}} \Delta_{h}^{r+1} f(x)} \\
& ={ }^{\wedge} C_{1}+C_{2}+C_{3}
\end{aligned}
$$

From (2) we know $\left|C_{2}\right| \leq C A_{\sigma}^{*}(f)$

For lemma 2, when $f(x) \in C^{j}, \Delta_{h}^{r+1} f(x)=\left(\frac{-2 \pi}{\sigma}\right)^{j}(-1)^{s} \Delta_{h}^{r+1-j} f^{(j)}\left(\xi_{i}\right)$

Where $x+(1-s) h \leq \xi \leq x+(1-s+j) h$,hence:

$$
C_{3}=\frac{1}{2^{r+1}} \sum_{i=0}^{r+1-j}(-1)^{i}\left(\begin{array}{c}
r+1-j \\
i
\end{array}\right) \Delta_{h}^{j} f(x+(1+i-s) h)
$$




$$
\begin{aligned}
& =\frac{1}{2^{r+1}}(-1)^{s}\left(\frac{-2 \pi}{\sigma}\right)^{j} \sum_{i=0}^{r+1-j}(-1)^{i}\left(\begin{array}{c}
r+1-j \\
i
\end{array}\right) f^{(j)}(\xi) \\
& =O\left(\frac{1}{\sigma^{j}} \omega\left(f^{(j)}, \frac{1}{\sigma}\right)\right)
\end{aligned}
$$

Now ,let's estimate $C_{1}$, from formula (2) and lemma 1

$$
\begin{aligned}
\left|C_{1}\right| & =\left|\sum_{k \in Z}\left(f\left(x_{k}\right)-F\left(x_{k}\right)\right) \frac{1}{2^{r+1}} \sum_{i=0}^{r+1}\left(\begin{array}{c}
r+1 \\
i
\end{array}\right)\right| F_{k}^{(\sigma)}(x)+(-1)^{s+i} F_{k+l}^{(\sigma)}(x)||=O\left(A_{\sigma}^{*}(f)\right) \cdot O(1) \\
& =O\left(A_{\sigma}^{*}(f)\right)
\end{aligned}
$$

Combining $C_{1}, C_{2}, C_{3}$, theorem 1 is proved.

As an application of the theorem 1 ,we can easy get the theorem 2.

THEOREM 2 For any continuous function $f(x), \lim _{\sigma \rightarrow \infty} H_{\sigma}(f ; r, x)=f(x)$

is valid.

\section{Conclusion}

The signal should be subject to limited bandwidth, if it is reconstructed from sampling signal by using Whittaker-Shannon series. In the paper, we propose a new Whittaker-Shannon series $H_{\sigma}(f ; r, x)$, which could easy the restriction of reconstruction of signal, i.e., the signal is just subject to infinite derivations. In addition, the aliasing error between original signal and reconstructed one can be determined accurately. The rank of aliasing error equals $\mathrm{j}$ if the signal has the $\mathrm{j}$-th derivation. As a extension of the proposal, any signal could be reconstructed if only it's continuous

\section{References}

[1] R.Gervais,Q.I.Rahman,G.Schmeisser.Simultaneous interpolation and approximation[M]//Badri N.Sahney(ed.) Polynomial and Spline Approximation. Dordrwencht/ Boston : D.Reidel Publ.Co.,1979: 203 223

[2] F.Pinter.A Jackson-type estimation on the real line by interpolation.Acta Math.Hung[J]. 1987, 49(2): 241-252

[3] L.Szili,P.Vertesi On Uniform Convergence of Sequences of Certain Linear Operators,Acta Math.Hungar[J]. 2001, 91(4): 159-186

[4] L.Szili On the Summability of Trigonometric Interpolation Processes Acta Math.Hungar[J]. 2001 , 91(4): 131-158

[5] Jaiyoung Chung Sampling Theorem for Entire Functions of Exponential Growth[J].J.Mathematical Analysis and Applications2002, 265(6): 217-228

[6] Kamalesh Kumar Sharma,Shiv Dutt Joshi, signal reconstruction from the undersampled signal samples[J].Optics Communications2006，268(8): 245-252

[7] M.H.Annaby,J.Bustoz,M.E.H.Ismail On sampling theory and basic Sturm-Liouville systems J.Commputational and Applied Mathematics[J]. 2007, 206(2): 73-85

[8] Tiber K.Pogany, Whittaker-type derivative sampling reconstruction of stochastic L $\alpha(\Omega)$-processes[J].Applied Mathematics and Computation. 2007, 187(3): 384-394

[9] Butzer P L,Higgins J R,Stens R L.Classical and approximate sampling theorems; studies in the $L^{\mathrm{p}}(\mathrm{R})$ and the uniform norm. J. Approx.Theory, 2005,137(3): 250-263

[10] Sun W. Density of wavelet frames. Appl. Comput. Anal. 2007，22(4): 264-272 